\title{
Analysis and Prediction of Collateral Channels from Left Superior Vena Cava after Bilateral Bidirectional Cavopulmonary Anastomosis
}

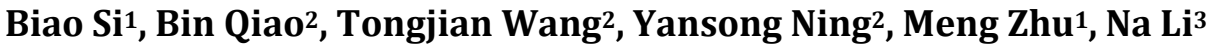 \\ ${ }^{1}$ Department of Medical Imaging, Institute of Cardiovascular Disease, General Hospital of Jinan Military Region, Jinan, China \\ ${ }^{2}$ Department of Cardiovascular Surgery, Institute of Cardiovascular Disease, General Hospital of Jinan Military Region, Jinan, \\ China \\ ${ }^{3}$ Medical Laboratory, Institute of Cardiovascular Disease, General Hospital of Jinan Military Region, Jinan, China \\ Email:wy3w@163.com
}

How to cite this paper: Si, B., Qiao, B., Wang, T.J., Ning, Y.S., Zhu, M. and Li, N. (2018) Analysis and Prediction of Collateral Channels from Left Superior Vena Cava after Bilateral Bidirectional Cavopulmonary Anastomosis. World Journal of Cardiovascular Diseases, 8, 237-247.

https://doi.org/10.4236/wjcd.2018.84023

Received: March 27, 2018

Accepted: April 27, 2018

Published: April 30, 2018

Copyright $\odot 2018$ by authors and Scientific Research Publishing Inc. This work is licensed under the Creative Commons Attribution International License (CC BY 4.0).

http://creativecommons.org/licenses/by/4.0/

\begin{abstract}
Objectives: To analyze and predict collateral channels in bilateral bidirectional cavopulmonary anastomosis focused on that from left superior vena cava. Methods: 41 consecutive patients who all underwent bilateral bidirectional cavopulmonary anastomosis were enrolled the study. The number, shape, size, origin and entry, and course of collateral vessels from left superior vena cava were referred for evaluation based on 41 cardiac angiographies. The influential factors on the formation of collateral vessels were identified by binary logistic regression analysis. Results: The number of the left superior vena cava with collateral vessels was larger than right one, 22 versus $8\left(X^{2}=10.303\right.$, $P=0.001)$. The medium-distal parts of left systemic vena were prone to development of collateral channel. The logic function with respect to the probability of left systemic venous collateral channel could be expressed as the linear expression of left systemic venous pressure $x: 0.418 x-7.111\left(X^{2}=\right.$ 23.095, $P<0.001)$. Setting $17.0 \mathrm{mmHg}$ of left systemic venous pressure as prediction cut-point to discriminate the occurrence of left collateral vessels, the predictive accuracy reached $85.7 \%$. Conclusions: The left systemic venous pressure was an independent factor associated with left systemic venous collateral channel after bilateral bidirectional cavopulmonary shunt. It might be practically feasible to predict the development of collateral vessels according to systemic venous pressure.
\end{abstract}

\section{Keywords}

Bilateral Bidirectional, Cavopulmonary Anastomosis, Glenn Procedure, Left Superior Vena Cava, Collateral Vessel 


\section{Introduction}

Bidirectional cavopulmonary anastomosis, which acted as an intermediate stage for the final complete Fontan procedure or as final palliation in high-risk patients with uninventricular physiology, can validly improve the systemic oxygen saturation of patients and reduce the risk of one-stage total cavopulmonary connection. But, bilateral bidirectional cavopulmonary anastomosis should be considered if persistent left superior vena cava was found in patients. That is, persistent left superior vena cava was connected with left pulmonary instead of being ligated up especially when it communicated with superior vena cava insufficiently.

Bidirectional cavopulmonary anastomosis usually leads to the development of collateral channel between the superior vena cava with higher pressure and the inferior vena cava with the lower pressure for decompression of systemic venous pressure [1]. In some patients, systemic venous collaterals would cause lethal postoperative desaturation and must be remedied [2].

However, the researches on development of collateral vessels arising from left superior vena cava in bilateral bidirectional cavopulmonary anastomosis were seldom documented in literatures due to the low incidence of the deformity. The study intended to investigate the occurrence regularity and risk factor associated with systemic venous collateral vessels based on the early follow-up results on 41 patients, focusing on collaterals from left superior vena cava in bilateral bidirectional cavopulmonary anastomosis.

\section{Materials and Methods}

\subsection{Subjects}

The present study was approved by Internal Research Ethics Board Committee of our hospital. Patients' parents had signed a series of consent forms in term of the test. Consecutive 41 patients who underwent one-stage bilateral bidirectional cavopulmonary anastomosis were enrolled the study, 20 males and 21 females, median age 7.0 (interquartile range 4.0 to 9.5 ) years. The criteria were established to select cases with a) functional single ventricle or hypoplastic left heart syndrome, b) the data of postoperative cardiac angiography and catheterization, c) azygos veins ligated during operation and d) additional pulmonary blood flow which was maintained in the repaired main pulmonary with $5 \mathrm{~mm}$ of diameter of pulmonary valve orifice. The other clinical characteristics of patients were listed in Table 1.

\subsection{Data Collection Methods}

On Allura CV20 unit (Philips Healthcare Andover, Massachusetts, USA) with St-smart imaging workstation (Crealife, Peking, China), total 41 times of cardiac catheterizations were performed. The static pressure of aorta, pulmonary artery and right ventricle were acquired by right heart technique while the static pressure of bilateral superior vena cava was monitored through jugular vein cannula. 
Table 1. Case characteristics.

\begin{tabular}{|c|c|c|}
\hline Items & Available pts. & Value \\
\hline Age at operation (years) & 41 & $5.0(3.0$ to 8.5$)$ \\
\hline Age at study (years) & 41 & $7.0(4.0$ to 9.5$)$ \\
\hline Weight at surgery $(\mathrm{Kg})$ & 41 & $15.5(13.0$ to 22.0$)$ \\
\hline Weight at study $($ Kg) & 41 & $16.5(14.5$ to 23.0$)$ \\
\hline Arterial oxygen saturation pre surgery (\%) & 39 & $75.2 \pm 10.4$ \\
\hline Arterial oxygen saturation at study (\%) & 41 & $87.4 \pm 5.8$ \\
\hline Interval since surgery to study (days) & 41 & $230.0(106.0$ to 379.0$)$ \\
\hline Hospital stay (days) & 40 & $12.0(10.0$ to 18.0$)$ \\
\hline Ventilator assistance duration (hours) & 40 & $5.0(4.0$ to 6.5$)$ \\
\hline Chest drain duration (days) & 40 & $3.0(3.0$ to 4.0$)$ \\
\hline ICU stay (days) & 40 & $4.0(3.0$ to 5.0$)$ \\
\hline Hematocrit pre surgery $(\%)$ & 40 & $63.0(52.5$ to 68.3$)$ \\
\hline Hemoglobin pre surgery $(g / L)$ & 40 & $197.0(169.0$ to 210.0$)$ \\
\hline Hematocrit at study (\%) & 36 & $46.3 \pm 5.7$ \\
\hline Hemoglobin at study $(g / L)$ & 41 & $145.5 \pm 18.9$ \\
\hline Pressure at left pulmonary artery $(\mathrm{mmHg})$ & 39 & $15.0(12.0$ to 20.0$)$ \\
\hline Pressure at right pulmonary artery $(\mathrm{mmHg})$ & 37 & $15.9 \pm 4.5$ \\
\hline Pressure at left caval vein $(\mathrm{mmHg})$ & 41 & $17.0(12.0$ to 23.0$)$ \\
\hline Pressure at right caval vein $(\mathrm{mmHg})$ & 40 & $15.0(13.0$ to 20.8$)$ \\
\hline Pulmonary vascular resistance ( Wood) & 27 & $4.1 \pm 2.2$ \\
\hline Pulmonary circulatory volume $(\mathrm{L} / \mathrm{min})$ & 27 & $3.9(2.2$ to 4.9$)$ \\
\hline
\end{tabular}

Pearson's Chi-square test is used to determine if the frequency of systemic venous collaterals is different between various originating position on caval vein, various draining pathways and various diameters. The numbers in the parentheses in 3th column are frequency of left systemic venous collaterals. $P$ values $<0.05$ were considered statistically significant. RSVC right superior vena cava, LSVC left superior vena cava.

The resistance and the volume of pulmonary circulation were calculated from data of pressure monitoring and blood gas analysis based on thermodilution theory.

The morphological observation for superior vena cava, cavopulmonary anastomosis and collateral vessels was undertaken by employing two experienced senior physicians with blinding to test objective. Main assessment aspects involved: the patency of systemic vena and cavopulmonary anastomosis; the number, shape, size, origin and entry, and course of collateral vessels. Each systemic vena was artificially divided into three parts. Two equidistantsegments of systemic vena between anastomotic stoma and originating site of subclavian vein were assumed to be proximal and medium vena cava, while the remainder was regarded as distal part extending cranially. The systemic vena $<80 \%$ of the local pulmonary in diameter was defined as vascular stenosis while $>120 \%$ as vascular 
dilatation, with or without distortion. The presence of collateral vessels was confirmed if reported concurrently by two observers. With respect to originating site and drainage destination of collateral vessels, definite agreement was reached by consultation.

Clinical data were collected conventionally.

\subsection{Statistical Analyses}

Retrospective statistical analyses on the data of cardiac catheterization were performed in SPSS 19.0 (SPSS, Chicago, Illinois, USA). Sample size depended on existing cases. The data in normal distribution were reported as mean \pm one standard deviation, or else as median with interquartile range. Categorical data were summarized as contingency table, and their relevance to occurrence of systemic venous channel was determined by Pearson's Chi-square test. Being prescreened on the basis of univariate hypothesis test, the influential factors on the formation of collateral vessels were identified by using binary logistic regression analysis. Forward method in the building of a multivariable logistic regression model was adopted by setting entry and removal criterion as 0.05 of probability in software. Receiver operating characteristic curve was employed for evaluating the fitness of binary logistic regression equation. Statistical significance was determined in all process at a two-tailed $P$ value of less than 0.05 .

\section{Results}

The occurrence of bilateral anatomic abnormality was statistically significant difference $\left(X^{2}=5.959, P=0.015\right) .24$ out of 41 left caval veins presented structure abnormality with not only vascular stenosis (12/41) but also dilatation $(12 / 41)$.On the contrary, only vascular dilatation (13/41) was found on right caval veins. All the vascular stenosis appeared adjacent to anastomotic stoma, and all the distention of vessel occurred at proximal part of systemic venous above cavopulmonary anastomosis. An example imaging was showed in Figure 1.

Systemic venous collateral channels were found on 30 systemic venae of 27 patients, with total 35 originating sites on vessels and 39 draining pathways. Total incidence of caval collaterals was $65.9 \%$ (27/41). The drainage course of collaterals showed statistically significance difference $\left(X^{2}=31.827, P<0.001\right)$, and $61.5 \%(24 / 39)$ of collaterals drained into the inferior vena cava via pericardiophrenic and internal thoracic veins (Table 2).

The number of the left superior vena cava with collateral vessels was larger than right one, 22 versus $8\left(X^{2}=10.303, P=0.001\right) .27$ out of total 35 originating site of systemic venous channel occurred on left superior vena cava. The frequency distribution of systemic venous collaterals is statistically significant different on various parts of systemic vena $\left(X^{2}=33.497, P<0.001\right)$, and them edium-distal part of left systemic vena were prone to development of collateral channel (Table 2, also Figure 1). 


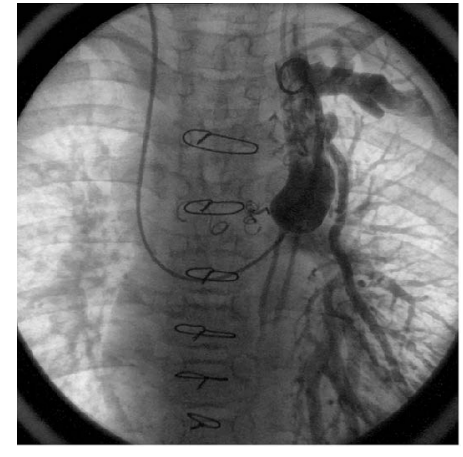

(a)

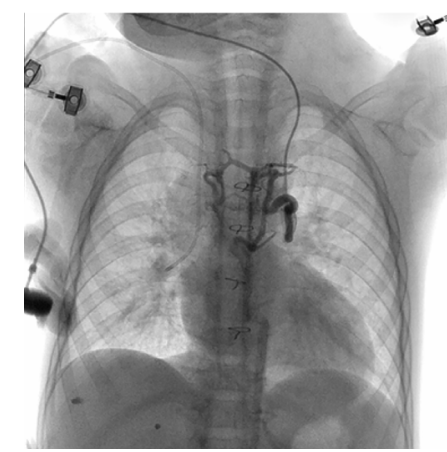

(c)

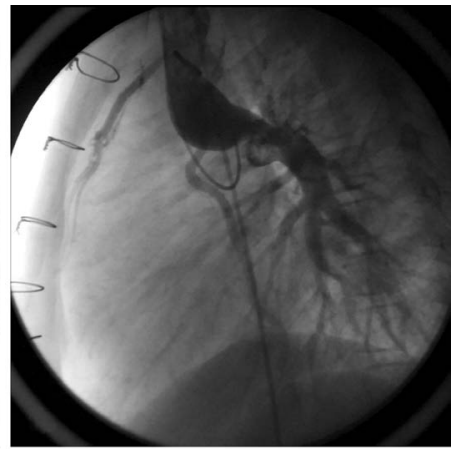

(b)

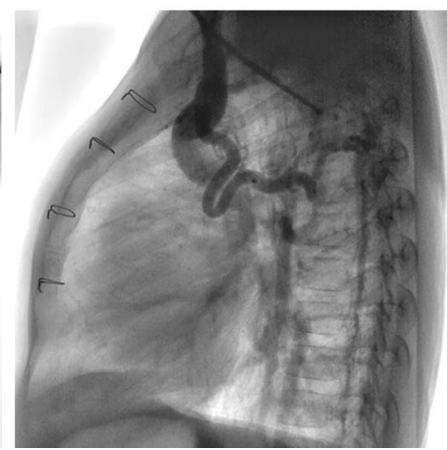

(d)

Figure 1. Angiogram example of systemic venous collateral channel. (a) (b) Angiogram of left caval vein on a patient who suffered from functional single ventricle and underwent bilateral cavopulmonary anostosis at 7 years old. Two notable collateral vessels were found, which originated from dilated caval vein accompanied with anastomotic stricture. One drained blood into inferior vena cava via pericardiophrenic vein in anterior mediastinum, the other via internal thoracic vein in mediastinum medium (upper panel); (c) (d) Another case with systemic venous collateral channel after surgery at five years old. azygous venous system and paravertebral venous plexus were main drainage pathway (lower panel).

Preliminary analysis for influential factor associated with the occurrence of systemic venous collaterals by univariate logistic regression methods summarized in Table 3.

The logic function with respect to the probability of left collateral vessels occurrence could be expressed as the linear regression expression of left systemic venous pressure:

$$
\ln \left(\frac{P}{1-P}\right)=0.418 x-7.111
$$

with statistical significance on regression model fitness $\left(X^{2}=23.095, P<0.001\right)$, where $P$ in the expression was the probability of left collateral occurrence and $x$ denoted the pressure of left superior vena cava. Left superior vena cava pressure was the major influencing factors on collateral angiogenesis with 1.519 of odds ratio (95\% CI: 1.167 to 1.977 ). Setting the cut-point of 0.5 as prediction probability which was corresponding to $17.0 \mathrm{mmHg}$ of left systemic venous pressure, the predictive accuracy reached $85.7 \%$ when discriminated whether the left 
Table 2. Frequency distribution of systemic venous collaterals.

\begin{tabular}{|c|c|c|c|c|}
\hline Items & Details & Numbers & Chi-square & $P$ value \\
\hline \multirow{6}{*}{ Collateral originating site $(n=35)$} & Distal RSVC & 1 & \multirow{6}{*}{33.497} & \multirow{6}{*}{$<0.001$} \\
\hline & Middle RSVC & 4 & & \\
\hline & Proximal RSVC & 3 & & \\
\hline & Distal LSVC & 12 & & \\
\hline & Middle LSVC & 14 & & \\
\hline & Proximal LSVC & 1 & & \\
\hline \multirow{8}{*}{ Collateral draining pathway $(n=39)$} & Azygous venous system & (4) 5 & \multirow{8}{*}{31.827} & \multirow{8}{*}{$<0.001$} \\
\hline & $\begin{array}{l}\text { Paravertebral venous } \\
\text { plexus }\end{array}$ & (1) 1 & & \\
\hline & Pericardiophrenic vein & (9) 12 & & \\
\hline & Mediastinal venous rete & (1) 1 & & \\
\hline & Internal thoracic vein & (9) 12 & & \\
\hline & Superior intercostal vein & (1) 1 & & \\
\hline & Cervical venous plexus & (4) 5 & & \\
\hline & coronary sinus & (1) 2 & & \\
\hline \multirow{4}{*}{ Collateral size $(n=39)$} & $\leq 1 \mathrm{~mm}$ & (6) 9 & \multirow{4}{*}{32.103} & \multirow{4}{*}{$<0.001$} \\
\hline & $2-7 \mathrm{~mm}$ & (17) 22 & & \\
\hline & $\geq 8 \mathrm{~mm}$ & (6) 7 & & \\
\hline & venous plexus & (1) 1 & & \\
\hline
\end{tabular}

Pearson's Chi-square test is used to determine if the frequency of systemic venous collaterals is different between various originating position on caval vein, various draining pathways and various diameters. The numbers in the parentheses in 3th column are frequency of left systemic venous collaterals. $P$ values $<0.05$ were considered statistically significant. RSVC right superior vena cava, LSVC left superior vena cava.

collateral vessels would occur. The predictive accuracy of the test was investigated by receiver operating characteristic analysis in Figure 2 .

\section{Discussion}

The incidence of persistent left superior vena cava in congenital heart disease was approximatively $3 \%-5 \%$ [3]. Persistent left superior vena cava mainly drains into the right atrium through a dilated coronary sinus [4]. However, if it drains directly into pulmonary veins or left atrium, a right-to-left shunt would be occurred [5]. It was crucial to recognize this anomaly when Glenn shunt or Fontan procedure is adopted for correcting functional single ventricle. For patients with persistent left superior vena cava, if only right superior vena cava was anastomosed with pulmonary artery, the notable blood from the upper body drained back to the atrium through left superior vena cava, especially when the communication between bilateral superior vena cava was poor. Without blood-oxygen exchange, this part of blood would result in a decrease of effective volume of pulmonary circulation and an increase of ventricular volume load. To 
Table 3. Preliminary analysis for influential factor associated with the occurrence of systemic venous collaterals by univariatelogistic regression methods.

\begin{tabular}{clll}
\hline $\begin{array}{c}\text { Factors involved in univariatelogistic } \\
\text { regression }\end{array}$ & Score & DF & $P$ Value \\
\hline Hemoglobin at study & 0.001 & 1 & 0.976 \\
ICU stay & 0.015 & 1 & 0.903 \\
Age at study & 0.091 & 1 & 0.763 \\
Ventilator assistance duration & 0.197 & 1 & 0.657 \\
Age at operation & 0.384 & 1 & 0.536 \\
Pulmonary circulatory volume & 0.395 & 1 & 0.530 \\
Hemoglobin pre surgery & 0.625 & 1 & 0.429 \\
Weight at study & 0.686 & 1 & 0.408 \\
Chest drain duration & 0.690 & 1 & 0.406 \\
Weight at surgery & 0.696 & 1 & 0.404 \\
Hematocrit at study & 0.720 & 1 & 0.396 \\
Arterial oxygen saturation at study & 0.920 & 1 & 0.337 \\
Hematocrit pre surgery & 0.937 & 1 & 0.333 \\
Arterial oxygen saturation pre surgery & 1.670 & 1 & 0.196 \\
Interval since surgery to study & 2.436 & 1 & 0.119 \\
Gender & 2.485 & 1 & 0.115 \\
Pressure at left caval vein & 3.243 & 1 & 0.072 \\
Time of hospital stay & 4.007 & 1 & 0.045 \\
\hline venous malfamation & 17.379 & 1 & $<0.001$ \\
\hline
\end{tabular}

The influential factors on the formation of collateral vessels were prescreened by using univariate logistic regression methods. The factors of $P$ values $<0.4$ in score test for regression model (marked as bold) were considered enter multivariable logistic regression analysis.

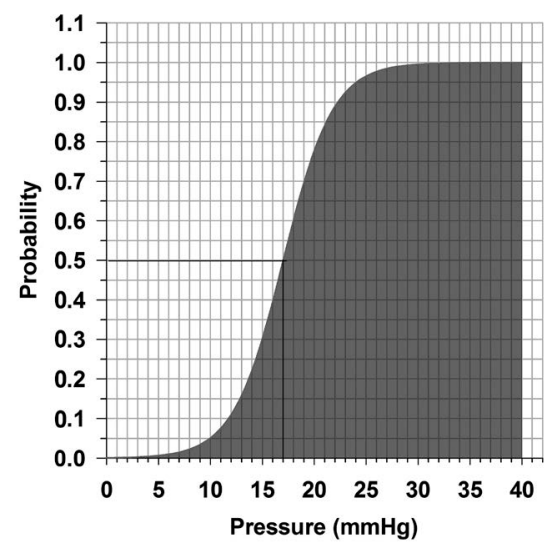

(a)

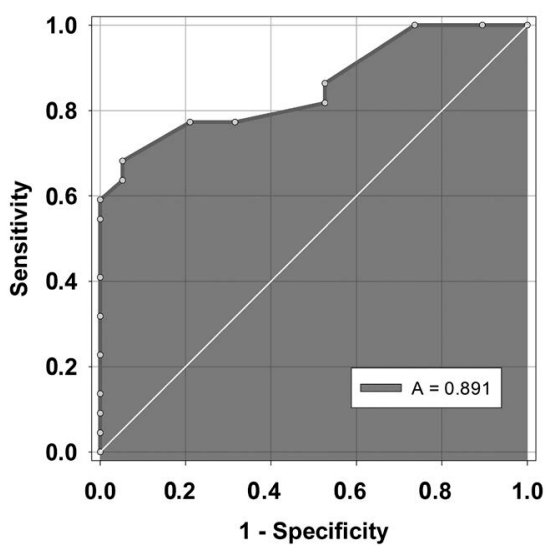

(b)

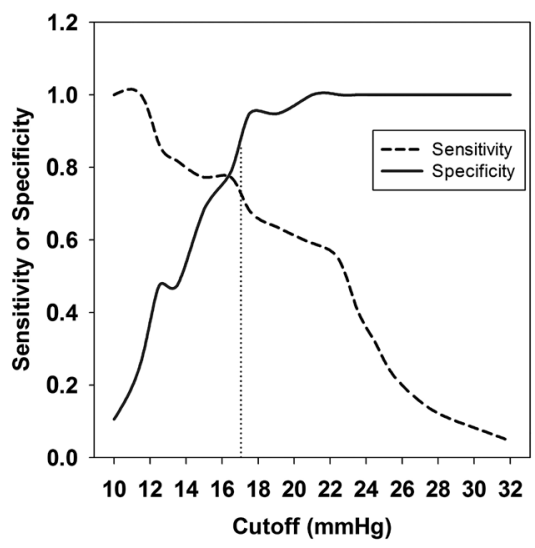

(c)

Figure 2. Logistic regression analysis. (a) Relationship between the pressure of left superior vena cava and the occurrence probability of systemic venous collateral. It results from binary logistic regression; (b) Receiver operating characteristic curve for evaluating the fitness of binary logistic regression equation. Area under the curve is 0.891 , and $P$ value is below 0.001 as null hypothesis is that true area $=0.5$; (c) Available prediction cutoff of left superior vena cava pressure in receiver operating characteristic analysis. Optimal sensitivity and specificity are obtained while cutoff value is $17 \mathrm{mmHg}$. The point was closer to the top-left part of the receiver operating characteristic curve. 
general opinion, persistent left superior vena cava should be handled either by ligating when it was fine and has sufficient communication with right vena cava, or by performing bilateral bidirectional cavopulmonary anastomosis when it was large enough to match with pulmonary artery in size.

The study showed that the occurrence of anatomic abnormality of left superior vena cava and cavopulmonary anastomosis was higher than that in right structure, and collateral vessels tended to occur on left systemic vein. Several possible reasons accounted for these results. First of all, the most vital thing that should be mentioned was about leaving additional pulmonary blood flow in Glenn shunt. It could inevitably increase the pressure in superior vena cava [6]. Anwar and his colleagues had reported that additional pulmonary blood flow through the main pulmonary trunk was inclined to flow into the left lung, which aggravated the burden on left lung [7]. Besides, pulmonary vascular bed was smaller in the left lung of two lobes than in the right lung of three lobes. The left lung presented with more weaken compensatory ability to adapt to sudden change of the vessel reconstruction by surgery with relatively higher pulmonary resistance. Furthermore, when persistent left superior vena cava developed insufficiently compared with superior vena cava, it was mismatched with the left pulmonary artery both in diameter and impedance [8]. As reasons mentioned above, it could be deduced that the flow in left superior vena cava would be slower. Based on Bernoulli equation, the slower flow can contribute to increasing hydrostatic pressure which might be an independent risk factor related to the presence of collateral vessels.

Our results showed that the occurrence of systemic venous collaterals following bilateral cavopulmonary anastomosis (65.9\%) was significantly higher than that in the priorstudy either on cavopulmonary anastomosis (33\%) or on Fontan procedure (20.2\%) [9] [10]. It implied the difference in circulatory physiology between cavopulmonary anastomosis with and without left super vena cava. Maybe, some other fluid dynamics mechanism not involved in the study could better explain it. Some impact on collaterals development, such as unbalanced pulmonary blood distribution, "liver factor" and weaken-pulsatile flow in superior vena cava, should be further investigated intensively by large samples study in future.

For patients who suffered from functional single ventricle, Salim and his associates reported that the proportion of the superior vena cava blood accounted for total cardiac output was about $55 \%-60 \%$ when $2-3$ years of age, then gradually decreased to $35 \%$ up to about 6 years old [11]. Thus, on Glenn shunt, reduced blood in superior vena cava directly leaded to a reduction of effective pulmonary volume with age until pulmonary circulation resistance tended to be stable. Moreover, systemic venous collaterals might partially decompress the elevated pressure in the systemic venous subsequent to completion of cavopulmonary anastomosis. It implied a steady tendency of development of venous collaterals. But on the other hand, pulmonary vessels growth appears retarded, and hypoxia condition seems to be deteriorative with patients grew up. Consequently, patho- 
logical changes in pulmonary small arteries might irreversibly lead to an increase of pulmonary resistance and static pressure that promoted the formation of venous collaterals. In summary, the vena cava collaterals might increase over time especially on patients who underwent operation after 6 years old. Unfortunately, because of very short follow-up period, we had no evidence to convince the fact that the interval since surgery to study was the influential factors associated with the development of collateral channels.

It was difficult for Doppler ultrasound to get a clear image of collateral vessels because echo intensity was prone to disturbance by air in lung fields and because available sound/blood angle was hard to obtain [12]. We proposed tentatively simple way to predict the occurrence of collateral vessels derived from left superior vena cava in bilateral bidirectional cavopulmonary anastomosis based on left superior vena cava pressure. The predictive accuracy reached $85.7 \%$ when set $17.0 \mathrm{mmHg}$ of left superior vena cava pressure to determine whether the systemic venous collaterals were present. If the predictive accuracy was verified by a mount of practical results later, this method would provide an objective basis for surgeons to prescribe further treatment on patients. Because central venous pressure was easy obtained in care unit, it must be practically feasible to predict the development of collateral vessels according systemic venous pressure. However, we did not obtain similar relationship between right systemic venous pressure and right collaterals occurrence. It might be supposed that systemic venous pressure was not higher enough to be a risk factor of collaterals formation.

Several limitations would weaken the accuracy of the results as we anticipated. Firstly, potential confounding factors were not mentioned in the study, such as severity of illness, physical state of patients, etc. Moreover, small samples in the study did not completely cover all the type of collaterals drainage channel. It would seriously restrict the variables selection in binary logistic regression analysis. Another limitation was high operation age of patient cohort in study. However, it was commonly opinion that bidirectional cavopulmonary anastomosis should be performed early in life in order to provide early volume unloading [13].Last but not least, the work did not investigate the long-term effect of systemic venous collaterals flow on patients because of retrospective design. The associations between potential risk factors and the development of collaterals were required to be illustrated in future.

\section{Conclusion}

The work supplements the knowledge of systemic venous collaterals arising from left superior vena cava in bilateral bidirectional cavopulmonary anastomosis. It would benefit surgeons to accurately understand and identify the presence of venous collateral channels.

\section{References}

[1] Webber, S.A., Horvath, P., LeBlanc, J.G., Slavik, Z., Lamb, R.K., Monro, J.L., Reich, 
O., Hruda, J., Sandor, G.G. and Keeton, B.R. (1995) Influence of Competitive Pulmonary Blood Flow on the Bidirectional Superior Cavopulmonary Shunt. A Multi-Institutional Study. Circulation, 92, II279-II286.

https://doi.org/10.1161/01.CIR.92.9.279

[2] Gatzoulis, M.A., Shinebourne, E.A., Redington, A.N., Rigby, M.L., Ho, S.Y. and Shore, D.F. (1995) Increasing Cyanosis Early after Cavopulmonary Connection Caused by Abnormal Systemic Venous Channels. British Heart Journal, 73, 182-186. https://doi.org/10.1136/hrt.73.2.182

[3] de Leval, M.R., Ritter, D.G., McGoon, D.C. and Danielson, G.K. (1975) Anomalous Systemic Venous Connection. Surgical Considerations. Mayo Clinic Proceedings, 50, 599-610.

[4] Pai, R.G. (1999) Echocardiographic Features of Persistent Left Superior Vena Cava. Echocardiography, 16, 435-436. https://doi.org/10.1111/j.1540-8175.1999.tb00088.x

[5] Duymus, M., Yesilkaya, Y., Orman, G., Bozkurt, A. and Yilmaz, O. (2012) Persistent Left Superior Vena Cava Draining to the Left Atrium: A Case Report and Review of the Literature. Polish Journal of Radiology/Polish Medical Society of Radiology, 77, 65-66. https://doi.org/10.12659/PJR.883632

[6] Chen, Q., Tulloh, R., Caputo, M., Stoica, S., Kia, M. and Parry, A.J. (2015) Does the Persistence of Pulsatile Antegrade Pulmonary Blood Flow Following Bidirectional Glenn Procedure Affect Long Term Outcome? European Journal of Cardio-Thoracic Surgery: Official Journal of the European Association for Cardio-Thoracic Surgery, 47, 154-158; Discussion 158.

[7] Anwar, S., Whitehead, K.K. and Harris, M.A. (2014) Leaving Antegrade Pulmonary Blood Flow Results in Reversal of Flow in the Azygous Vein and Decompression of the Cavopulmonary Circulation: Mechanism of Cyanosis Defined with Cardiac Magnetic Resonance Imaging. Cardiology in the Young, 24, 515-516. https://doi.org/10.1017/S104795111300214X

[8] Povoski, S.P. and Khabiri, H. (2011) Persistent Left Superior Vena Cava: Review of the Literature, Clinical Implications, and Relevance of Alterations in Thoracic Central Venous Anatomy as Pertaining to the General Principles of Central Venous Access Device Placement and Venography in Cancer Patients. World Journal of Surgical Oncology, 9, 173. https://doi.org/10.1186/1477-7819-9-173

[9] McElhinney, D.B., Reddy, V.M., Hanley, F.L. and Moore, P. (1997) Systemic Venous Collateral Channels Causing Desaturation after Bidirectional Cavopulmonary Anastomosis: Evaluation and Management. Journal of the American College of Cardiology, 30, 817-824. https://doi.org/10.1016/S0735-1097(97)00223-4

[10] Weber, H.S. (2001) Incidence and Predictors for the Development of Significant Supradiaphragmatic Decompressing Venous Collateral Channels Following Creation of Fontan Physiology. Cardiology in the Young, 11, 289-294.

https://doi.org/10.1017/S1047951101000312

[11] Salim, M.A., DiSessa, T.G., Arheart, K.L. and Alpert, B.S. (1995) Contribution of Superior Vena Caval Flow to Total Cardiac Output in Children. A Doppler Echocardiographic Study. Circulation, 92, 1860-1865. https://doi.org/10.1161/01.CIR.92.7.1860

[12] Yano, S. and Shimada, K. (1997) Changes in Superior Vena Cava Pulsed Doppler Flow Patterns: Possible Indicator of Improvement of Superior Vena Cava Syndrome Due to Lung Cancer. Journal of Ultrasound in Medicine: Official Journal of the American Institute of Ultrasound in Medicine, 16, 707-710. https://doi.org/10.7863/jum.1997.16.10.707 
[13] Gerelli, S., Boulitrop, C., Van Steenberghe, M., Maldonado, D., Bojan, M., Raisky, O., Sidi, D. and Vouhe, P.R. (2012) Bidirectional Cavopulmonary Shunt with Additional Pulmonary Blood Flow: A Failed or Successful Strategy? European Journal of Cardio-Thoracic Surgery. Official Journal of the European Association for Cardio-Thoracic Surgery, 42, 513-519. https://doi.org/10.1093/ejcts/ezs053 